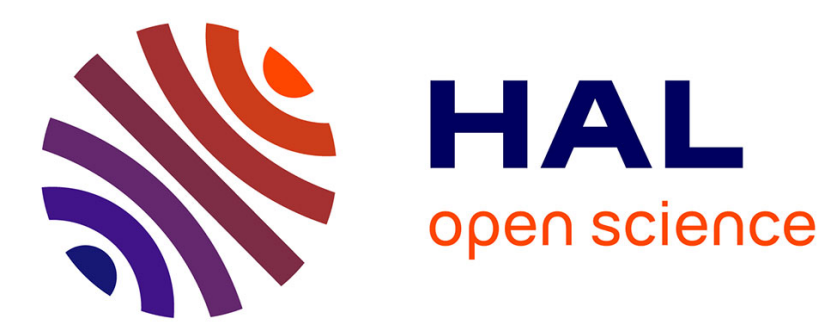

\title{
Optimisation of Preform Temperature Distribution For the Stretch-Blow Moulding of PET Bottles
}

\author{
Maxime Bordival, Yannick Le Maoult, Fabrice Schmidt
}

\section{To cite this version:}

Maxime Bordival, Yannick Le Maoult, Fabrice Schmidt. Optimisation of Preform Temperature Distribution For the Stretch-Blow Moulding of PET Bottles. International Journal of Material Forming, 2008, 1 (Suppl 1), pp.1023-1026. 10.1007/s12289-008-0232-8 . hal-01709503

\section{HAL Id: hal-01709503 \\ https://hal.science/hal-01709503}

Submitted on 19 Mar 2019

HAL is a multi-disciplinary open access archive for the deposit and dissemination of scientific research documents, whether they are published or not. The documents may come from teaching and research institutions in France or abroad, or from public or private research centers.
L'archive ouverte pluridisciplinaire HAL, est destinée au dépôt et à la diffusion de documents scientifiques de niveau recherche, publiés ou non, émanant des établissements d'enseignement et de recherche français ou étrangers, des laboratoires publics ou privés. 


\title{
Optimisation of Preform Temperature Distribution For the Stretch-Blow Moulding of PET Bottles
}

\author{
M. Bordival, Y. Le Maoult, F.M. Schmidt \\ CROMeP, Ecole des Mines Albi, Campus Jarlard, 81000 Albi, France
}

\begin{abstract}
This study presents an optimization strategy developed for the stretch-blow moulding process. The method is based on a coupling between the Nelder-Mead optimization algorithm, and Finite Element (FE) simulations of the forming process developed using ABAQUS®. FE simulations were validated using in situ tests and measurements performed on $18.5 \mathrm{~g}-50 \mathrm{cl}$ PET bottles. To achieve that, the boundary conditions were carefully measured for both the infrared heating and the blowing stages. The temperature distribution of the perform was predicted using a 3D finite-volume software, and then applied as an initial condition into FE simulations. Additionally, a thermodynamic model was used to predict the air pressure applied inside the preform, taking into account the relationship between the internal air pressure and the enclosed volume of the preform, i.e. the fluid-structure interaction. It was shown that the model adequately predicts both the blowing kinematics and the thickness distributions of the bottle. In a second step, this model was combined to an optimization loop to automatically compute the best perform temperature distribution, providing a uniform thickness for the bottle. Only the last part will be fully detailed in this paper.
\end{abstract}

Key words: Stretch-blow moulding, PET bottles, heat transfer, finite element method, optimization.

\section{INTRODUCTION}

Among the techniques devoted to the manufacture of PolyEthylene Terephtalate (PET) bottles, the twostage Stretch-Blow-Moulding (SBM) process is probably the most popular. This process involves the manufacture of structurally amorphous semiproducts, called preforms, made by injection moulding of PET resin. A reheating step is necessary to heat the preforms to the appropriate temperature distribution above the glass transition, which is typically around $80^{\circ} \mathrm{C}$ for PET. This stage is generally performed using infrared (IR) heaters, taking advantage of the semitransparent behaviour of PET submitted to IR radiation. In a second stage, the preforms are stretched using a cylindrical rod, and blown using two levels of air pressure. Then, the bottles are cooled down by a mould whose temperature is regulated using cooling channels.
The heating conditions, that control the preform temperature distribution, strongly affect the blowing kinematics (stretching and inflation), and consequently the thickness distribution of the bottle. Temperature also affects the orientation induced by biaxial stretching, which in turn, affects mechanical, optical and barrier properties of bottles [1].

Regarding to BM, Lee and Soh [2] presented a FE optimization method to determine the optimal thickness profile of a preform, given the required wall thickness distribution for the blow-moulded part. More recently, Thibault et al. [3] proposed an automatic optimization of the preform geometry (initial shape and thickness) and operating conditions, using the nonlinear constrained algorithm Sequential Quadratic Programming (SQP). The robustness of the method was discussed through a comparison with experiments performed within industrial conditions. SQP was also used in order to optimize heating system parameters [4]. The 
objective was to homogenize the temperature along the preform length, by modifying the process parameters related to the IR oven. It is interesting to point out that authors questioned the relevance of the objective chosen for the optimization.

In this work, we propose a numerical optimization strategy for SBM. For that, we developed an iterative procedure allowing to automatically compute the best temperature distribution along the preform length, providing a uniform thickness for the bottle. We solve the optimization problem by coupling FE simulations to the Nelder-Mead optimization algorithm (nonlinear simplex). Results were validated by careful in situ tests and measurements performed on $18.5 \mathrm{~g}$ - 50cl PET bottles. To achieve that, special attention was given to the measurement of boundary conditions required for both the infrared heating stage, and the blowing stage.

\section{OPTIMIZATION OF PREFORM TEMPERATURE}

The performance of a bottle manufactured by SBM is drastically affected by its thickness distribution. In order to achieve bottles with appropriated thickness distributions, it is more desirable to adjust the process conditions, and to use the same design of preform for making different shapes of bottles. This approach aims to minimize the cost associated with the design of a new perform (especially the manufacture of a new injection mould). Determining adequate operating conditions remains nevertheless costly and time consuming. Different approaches are possible, such as trial-and error methods, or design of experiments. Both of them require a large number of experiments (or simulations), especially when the parameters are strongly interdependent. As a consequence, they become inadequate and impracticable for complex problems. In contrast, the optimization algorithms make the optimization process fully automatic, and from this point of view, yield a significant assist in the development cycle.

In this section, we propose to couple an optimization algorithm to FE simulations in order to optimize the temperature distribution along the preform length. The goal will be to provide an homogeneous thickness for the bottle. Infrared heating and blow moulding numerical models have been fully detailed in a previous paper [5]. A typical blow moulding simulation is presented in figure 1 where we compare the thickness distribution computed, and the thickness profile measured. Measurements were averaged on a set of three trials. We observe a good agreement along most part of the bottle (less than 15 $\%$ error on the mean thickness).

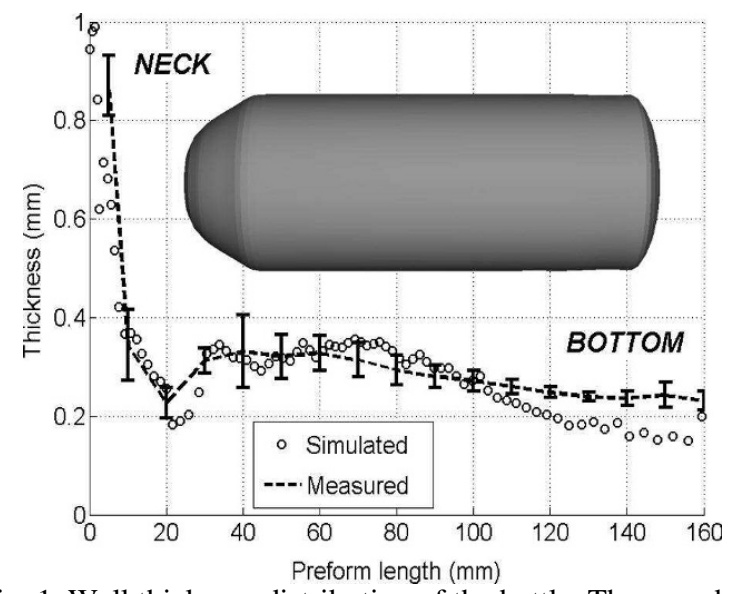

Fig. 1. Wall thickness distribution of the bottle. The error bars show \pm 1 standard deviation for a set of 3 trials.

\subsection{Parameterisation and constraints}

In order to describe the temperature distribution along the preform length, we consider three optimization variables. They correspond to three temperatures located at different heights of the preform, as illustrated by figure 2 .

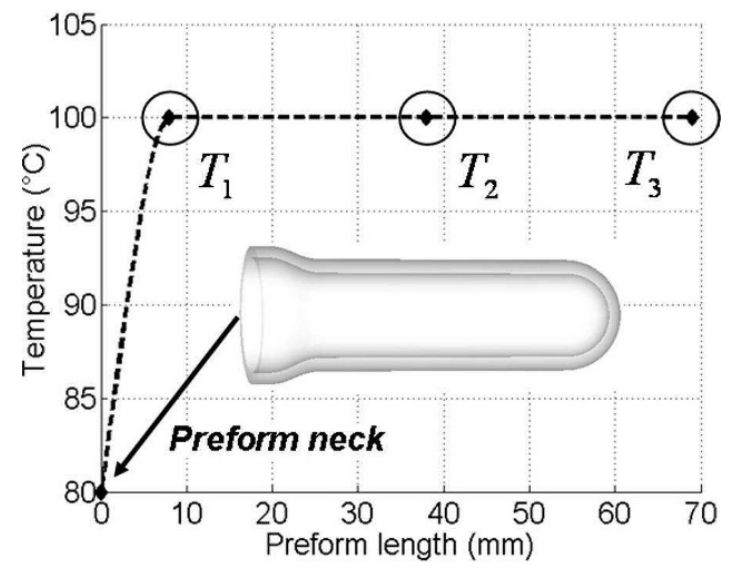

Fig. 2. Temperature distribution along the preform length Optimization variables.

The whole temperature distribution is then deduced using the Piecewise Cubic Hermite Interpolating Polynomial (PCHIP) method [6]. To provide an accurate interpolation, an additional temperature is added on the preform neck. This fourth temperature is not optimized, but fixed to $80^{\circ} \mathrm{C}$, which 
corresponds approximately to the glass transition of PET. Indeed, throughout the reheating stage, the preform neck is generally protected from IR radiation in order to prevent its temperature from exceeding the PET glass transition. This approach aims to prevent any deformation of the bottle neck during the forming process. Finally, to simplify the problem, the temperature is assumed to be uniform through the preform thickness. The optimization variables are constrained using lower and upper bounds, corresponding respectively to the PET glass transition temperature, and to the PET crystallization temperature. These two physical limits have been naturally chosen to prevent serious strengthening of the structure from appearing, in which case, any deformation would be proscribed during the forming stage. Let us note that neither linear nor nonlinear constraint is required.

\subsection{Objective function}

In this application, we attempt to provide a uniform thickness for the bottle. This objective must be mathematically formulated by an appropriate costfunction. A simple way to proceed is to define the objective function $F$ as the standard deviation of the computed thicknesses, as following:

$$
\mathrm{F}(\overrightarrow{\mathrm{x}})=\sqrt{\frac{1}{\mathrm{n}-1} \sum_{\mathrm{i}=1}^{\mathrm{n}}\left(\mathrm{th}_{\mathrm{i}}-\overline{\mathrm{th}}\right)^{2}}
$$

where $\vec{x}$ represents the set of optimization variables, $\mathrm{n}$ is the number of nodes along the bottle height, $\mathrm{th}_{\mathrm{i}}$ is the thickness at the node " $\mathrm{i}$ ", and $\overline{\mathrm{th}}$ is the mean thickness. The nodal thicknesses are computed using a Python script that we have developed into ABAQUS® FEM software. Such a function is null for a bottle with perfectly uniform thickness.

\subsection{Choice of an Algorithm}

The choice of the optimization algorithm is closely related to the type of cost function. In our application, we attempt to minimize a nonlinear realvalued function, subject to bound constraints. In addition, strong mechanical and geometric nonlinearities could induce significant numerical instabilities, making the objective-function noisy, and therefore non-differentiable. As a consequence, the gradient-based algorithms might not be adapted to this type of problem. In contrast, the direct search methods (which do not require the computation of the cost-function gradient) remain particularly adapted to the non-derivative optimization. Among this family of methods, the Nelder-Mead simplex algorithm is probably one of the most popular. However, this local method provides relatively slow convergence rates [7]. Nevertheless, when the derivatives can not be explicitly written, this method can save a significant amount of computation time compared to gradient-based methods. Indeed, the computation of the cost-function gradients can become strongly time consuming when they are approximated using the finite-difference method. This is particularly true when the number of optimization variable is large.

On the other hand, the Nelder-Mead simplex algorithm is restricted to unconstrained problems. In this work, we used the method proposed by Luersen et al. [8] in order to add bound-constraints into the Nelder-Mead simplex algorithm available in Matlab® .

\subsection{Results and discussion}

All numerical results reported in the sequel were obtained on a $2.8 \mathrm{GHz}-512$ Mo Pentium 4. Figure 3 displays the decrease of the objective-function value in terms of the number of optimization iterations.

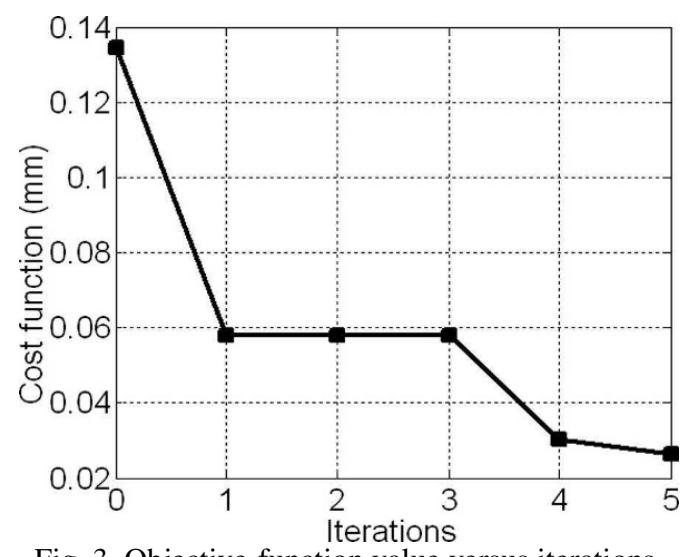

Fig. 3. Objective-function value versus iterations.

We observe that the objective function is reduced by $60 \%$ of its initial value after the first iteration, and by more than $80 \%$ at the end of the optimization process. Consequently, the thickness distribution of the formed bottle is $80 \%$ more uniformed after optimization. The algorithm converges after 5 iterations, which involves only 10 objective-function evaluations (that is to say, $10 \mathrm{FE}$ simulations). 
On average, one cost-function evaluation requires 26 min CPU. Thus, the total CPU time required for the optimization is approximately $3 \mathrm{~h} 20 \mathrm{~min}$. Figure 4 illustrates the temperature distribution along the preform length before and after optimization.

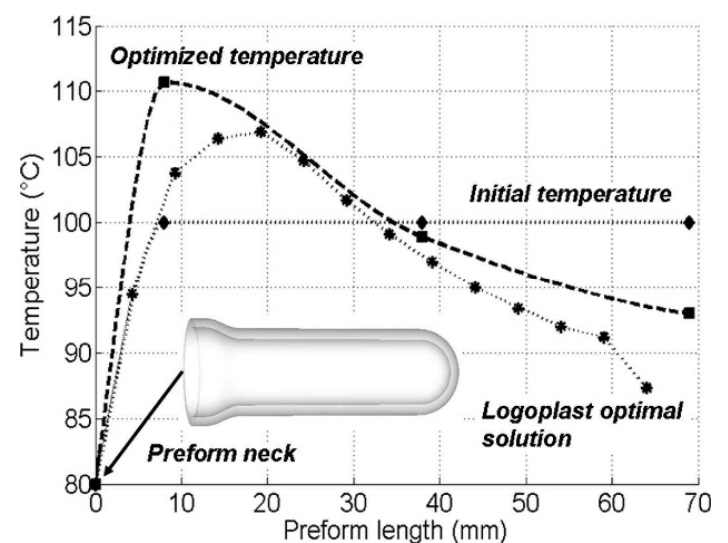

Fig. 4. Initial and optimized temperature distributions along the preform length.

Initial conditions were chosen in order to apply a uniform temperature $\left(100^{\circ} \mathrm{C}\right)$ on the preform. Such temperature distribution leads to a strongly nonuniform thickness distribution for the bottle, as illustrated by Fig. 5 .

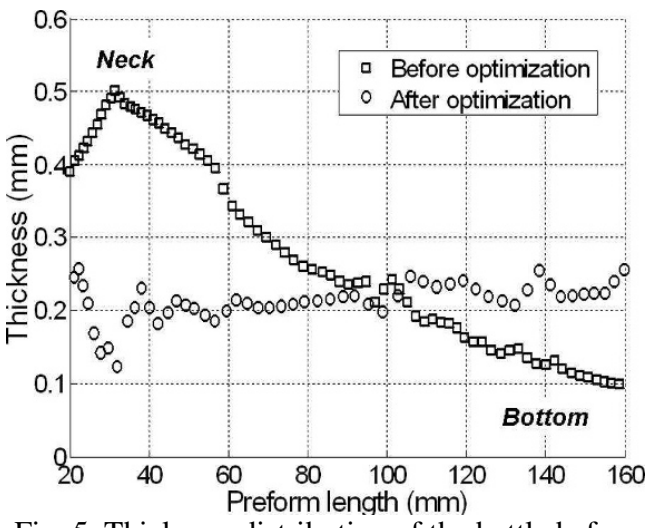

Fig. 5. Thickness distribution of the bottle before and after optimization.

After optimization, there is a temperature gradient along the perform length, which provides a more uniform thickness and a full blowing of the bottle. Fig. 4 also illustrates the optimal temperature distribution determined by Logoplast Company using an experimental trial-and-error method. This result has been obtained using the same preform, but with a different shape of mould. However, we can notice that there is a good agreement in the trends between the temperature profile experimentally determined within industrial conditions, and the temperature distribution computed using our optimization method.

\section{CONCLUSION}

For SBM optimization, we have proposed a practical methodology to numerically optimize the temperature distribution of a PET preform, in order to provide a uniform thickness for the bottle.

Encouraging preliminary results have shown the viability of our approach. However, it would probably be more desirable to directly optimize the process parameters of the heating systems. But to do so, both the infrared-heating simulation and the blowing simulation would need to be included into the optimization loop, resulting in further complications essentially due to long computation times.

Nevertheless, this approach would implicitly account for the influence of the temperature distribution through the preform thickness, which is of prime interest.

\section{ACKNOWLEDGEMENTS}

This study was conducted within the frame of 6th EEC framework. STREP project APT_pack; NMP - PRIORITY 3. www.apt-pack.com. Special thanks to Logoplast Technology for manufacturing the preforms, and QUB for their collaboration.

\section{REFERENCES}

1. G. Venkateswaran et al., Adv. Polym. Tech., 17, (1998).

2. D. K. Lee, S. K. Soh, Polym. Eng. Sci., 36, 11 (1996).

3. F. Thibault et al., Polym. Eng. Sci., 47, 3 (2007).

4. M. Bordival et al., Proc. of the Int. Conf. ESAFORM 9, p. 511-514 (2006).

5. M. Bordival et al., Proc. of the Int. Conf NUMIFORM 7, Porto, (2007)

6. F. N. Fritsch and R. E. Carlson, SIAM J. Numerical Analysis, 17, (1980).

7. J. C. Lagarias et al., SIAM J. Optimization, 9, 1 (1998).

8. M. A. Luersen and R. Le Riche, Proc. of the Int. Conf. on Eng. Comp. Tech., p 165-166 (2002). 